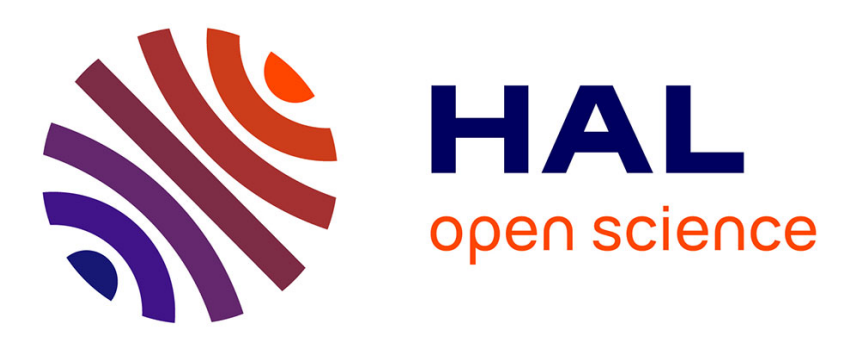

\title{
Towards the end of drying of granular materials: enhanced evaporation and drying-induced collapse
}

Zhongzheng Wang, Benjamin Maillet, Jean-Michel Pereira, Yixiang Gan

\section{To cite this version:}

Zhongzheng Wang, Benjamin Maillet, Jean-Michel Pereira, Yixiang Gan. Towards the end of drying of granular materials: enhanced evaporation and drying-induced collapse. Water Resources Research, 2021, 10.1029/2021WR030125 . hal-03271442

HAL Id: hal-03271442

https://hal-enpc.archives-ouvertes.fr/hal-03271442

Submitted on 25 Jun 2021

HAL is a multi-disciplinary open access archive for the deposit and dissemination of scientific research documents, whether they are published or not. The documents may come from teaching and research institutions in France or abroad, or from public or private research centers.
L'archive ouverte pluridisciplinaire HAL, est destinée au dépôt et à la diffusion de documents scientifiques de niveau recherche, publiés ou non, émanant des établissements d'enseignement et de recherche français ou étrangers, des laboratoires publics ou privés. 


\title{
Towards the end of drying of granular materials: enhanced evaporation and drying-induced collapse
}

\author{
Zhongzheng Wang, ${ }^{\mathrm{a}, \mathrm{b}}$, Benjamin Maillet $^{\mathrm{b}}$, Jean-Michel Pereira ${ }^{\mathrm{b}, *}$, Yixiang \\ $\operatorname{Gan}^{\mathrm{a}, \mathrm{c}, *}$ \\ ${ }^{a}$ School of Civil Engineering, The University of Sydney, NSW 2006, Australia \\ ${ }^{b}$ Navier, Ecole des Ponts, Univ Gustave Eiffel, CNRS, Marne-la-Valle, France \\ ${ }^{c}$ The University of Sydney Nano Institute, The University of Sydney, NSW 2006, Australia
}

\begin{abstract}
We experimentally study the drying of loosely packed wet glass beads at low initial water content. The drying rate is found to decrease at the start, corresponding to the decreasing rate period controlled by vapor diffusion, followed by a deviation in drying rate from the diffusion limited evaporation. The propagation of drying front associated with a sharp saturation gradient is identified through both image analysis and magnetic resonance imaging technique. The drying-induced collapse of granular medium is observed and quantified. The concentrated collapse at the end of drying suggests the existence of liquid in the form of liquid bridges in the apparent dry region until the end of drying process. Collapse event is found to be local, i.e., a clear boundary can be identified for each collapse event, below which the loosely packed medium remains intact. This indicates the existence of a saturation gradient in the apparent dry region. The drying dynamics and collapse statistics suggest that the observed transition of drying regimes is due to Kelvin effect. This work demonstrates for the first time the drying enhancement phenomenon due to Kelvin effect even for grains with size of hundreds of micrometers, and provides insights on the drying process of partially saturated granular materials, especially near the final period of evaporation.
\end{abstract}

\footnotetext{
${ }^{*}$ Corresponding author

Email addresses: jean-michel.pereira@enpc.fr (Jean-Michel Pereira), yixiang.gan@sydney.edu.au (Yixiang Gan)
} 
Keywords: Evaporation, drying, granular material, porous media, liquid bridge, MRI 


\section{Highlights}

- Drying-induced collapse of loosely packed granular media is observed and quantified.

- NMR technique is used to reveal the distribution of liquid during evaporation.

- The existence of liquid in the apparent dry region is evidenced by the concentrated collapse near the end of drying experiment.

- The enhancement of evaporation rate is observed and explained by Kelvin effect. 


\section{Introduction}

Drying of porous media is essential in relation with many applications, such as drugs and cosmetics synthesis in the pharmaceutical industry, thermally enhanced oil recovery, and drying of textiles, grains, and food. Particularly, soil drying is of great environmental importance, as it controls the water, energy and solutes transfer between the atmosphere and subsurface. The basic mechanisms of drying of saturated porous media have been identified as two distinguishable stages [1 8 ]. In the first stage, although the primary drying front (below which the medium is saturated) is receding into the medium, the evaporation of liquid at the surface is constantly being supplied through capillary flow, leading to a constant evaporation rate, which is limited by the external conditions (i.e, velocity and relative humidity of surrounding air), until either a critical surface water content [3] or a characteristic depth [5] is reached. Then, the evaporation enters the second stage, and the drying rate starts to decrease as a result of dominance of vapor diffusion, which is accompanied by the secondary drying front (separating the dry region and capillary flow region) receding into the porous media [2, 6 9]. A schematic showing the drying process of porous media is shown in Fig. 1. Efforts have been made to predict this transition mainly through the force balance among capillary, viscous, and gravitational forces, depending on properties of liquid and solid matrix, such as surface tension, viscosity, wettability, and pore size distribution [2, 3, 5 - 9 ]. Nevertheless, most existing studies focused on the drying process of initially saturated porous media, and the drying of partially saturated media with low liquid content, especially towards the extreme end of drying, remains relatively unexplored.

For porous media at low water content, i.e, in the pendular state [10 12], water exists mainly in the form of liquid bridges, making the granular material cohesive as a result of the capillary force from liquid bridges [10, 12 15]. As a result, studies have demonstrated a significant decrease in packing fraction of wet porous media compared with dry (or fully saturated) media [16 19]. This may have several implications for the dynamics of drying processes. Firstly, as 
reported in the literature, for cohesionless particles with size typically greater than $100 \mu \mathrm{m}$ (negligible van der Waals forces), the lower limit of packing fraction is around 0.55 (random very loose packing) 20-24]. Therefore, drying-induced collapse may occur in loosely packed wet granular materials (e.g., packing fraction less than 0.55 for mono-sized spheres) as the liquid bridges disappear during

45 evaporation. Besides, since the liquid bridges can be regarded as isolated in the pendular regime, the initial drying stage is expected to be fundamentally different from the saturated case where the connected liquid network can supply liquid towards the medium surface through capillary flow. Particularly, several studies have suggested that, apart from capillary flow, the film flow at grain surface due to adsorption can maintain the connectivity of liquid phase [25-28]. It is thus interesting to examine the potential influence of film flow on the drying process, especially at low initial water content.

In this work, we experimentally investigate the drying of loosely packed wet glass beads at low initial volumetric water content $(\sim 2 \%)$. Such low initial water

${ }_{55}$ is chosen in order to focus on the drying process near the end of drying, which also allows the generation of loosely packed granular assembly and investigation on the drying-induced collapse. The specimen was firstly prepared with desired packing fraction $(\sim 0.45)$ through a recently reported experimental procedure [11, 19], after which the drying experiment was conducted. The statistics of

60 collapse events during drying as well as the evolution of liquid distribution were obtained through image analysis and magnetic resonance imaging (MRI) technique, which provide global and local insights on physical mechanisms that govern the drying process.

\section{Experiments}

Materials and specimen preparation: Mono-sized glass beads of average diameter $375.5 \mu \mathrm{m}$ (standard deviation $31.8 \mu \mathrm{m}$ ) are chosen with consideration of (1) to exclude the effect from van der Waals forces on packing structure, and (2) to have significant decrease in packing fraction once water is added 
Figure 1: Schematic showing the drying process of porous media. In the constant rate period, evaporation of liquid at surface is supplied by capillary flow. After reaching a characteristic length $L^{*}$, the second drying front starts receding into the medium, marking the transition towards decreasing rate period. 
[11, 16 19]. The cumulative grain size distribution determined by laser diffraction technique (using Beckman Coulter Vsm+ Ls Variable Speed Fluid Module Plus) is shown in Fig. 2(b). The specimen was mixed thoroughly with water at volumetric water content $5 \pm 1 \%[11,19,29]$. Then, a sieve containing the wet glass beads was shaked by a vibration machine. A cylindrical container with inner diameter $13.70 \pm 0.01 \mathrm{~mm}$ and inner height $44.22 \pm 0.12 \mathrm{~mm}$ was placed under the sieve, which was supported by struts that are disconnected with the vibration machine. Wet grains fell progressively from the sieve into the container once the vibration starts. The free fall height of grains, i.e., the distance between the sieve and the container can be adjusted to control the resulting packing fraction [19]. After the specimen preparation, the initial volumetric water content and packing fraction were measured to be $w_{0}=1.7 \pm 0.2 \%$ and $\rho_{0}=0.45 \pm 0.01$, respectively. With the choice of grain and container size, which leads to a particle diameter-to-container diameter ratio smaller than 0.1, the boundary effect from the container wall can be neglected [19, 30].

Drying experiments: The specimens were dried at laboratory conditions at temperature of $21 \pm 1{ }^{\circ} \mathrm{C}$ and 0.55 relative humidity. The weight of each specimen was recorded every minute by an analytical balance (Sartorius CP224S) with nominal resolution of $0.1 \mathrm{mg}$, and photos were taken every 10 minutes by a camera (Canon 600D). Generally, the drying experiment under described conditions took around 45 hours to complete. The end of drying can be identified once the specimen weight stops changing and remains at a constant value with fluctuations of the resolution of balance (also indicated by the relatively sharp turning point of drying rate, e.g., in Fig. 2(a)). The completion of drying process was further verified by comparing the residual water content of specimen, calculated from the weight difference before and after heating in oven overnight 95 at $105{ }^{\circ} \mathrm{C}$, to that of dry specimen in laboratory condition, both of which were $0.029 \pm 0.001 \%$. The cumulative drift of balance was found to be less than 0.5 mg during the entire experiment.

MRI profiling experiment: The nuclear magnetic resonance imaging (MRI), as a non-invasive and non-destructive method, has proven effective 
in probing the evolution of saturation profile during the evaporation process [8, 31, 32]. Here, the drying experiments were also conducted to obtain the evolution of 1D saturation profile using MRI (Bruker Minispec mq20 equipped with a field gradient) under similar experimental conditions as a supplement to provide qualitative characterization of the drying process. Due to the limitais proportional to the total mass of water.

At the same time, we measured the NMR relaxation through a Carr-PurcellMeiboom-Gill [33] (CPMG) sequence composed of a first $\pi / 2$-pulse and 2500 $\pi$-pulses during $500 \mathrm{~ms}$ distributed in linear intervals. The repetition time is 2 130 tion on the size of NMR tube for the mini-spectrometer, the specimen size is smaller, i.e, inner diameter of $6.9 \mathrm{~mm}$ and inner height of $14.5 \mathrm{~mm}$. To remove the grains sticking at the inner wall, vibration is applied to the container such that grains on the wall fall down due to inertia. As a result of the extra vibration, the packing fraction of the granular assembly is around 0.58 for the MRI experiment, equal to the packing fraction of medium packed at dry condition, thus no collapse event is expected during the MRI experiment.

A multi-echo sequence with 128 echos was applied during the evaporation process. The echo time was $7.4 \mathrm{~ms}$, much smaller than the typical relaxation time of the water at $40 \mathrm{~ms}$ to avoid a $T_{2}$ weighting. The field of view was $20 \mathrm{~mm}$, greater than the specimen height. The number of pixels was 128, corresponding to a spatial resolution of $0.16 \mathrm{~mm}$. An averaging by 3 pixels was carried out to smooth the data. The recycle delay was $2 \mathrm{~s}$ (much greater than the total duration of the multi-echo sequence and typical value of the longitudinal relaxation time $T_{1}$ of the water in this specimen) to reach the magnetization equilibrium and to avoid warming of the specimen. The signal was accumulated 128 times in order to increase the signal-to-noise ratio, leading to the cycle period of about 4.3 minutes. A Fourier transform of each echo and a Gaussian filter were applied. An exponential fit of odd echos for each pixel was done to get the amplitude, which is proportional to the mass of water corresponding to each pixel. The integration of each 1D profile gives the total NMR signal, which

s for relaxation of protons. This sequence was repeated 128 times to increase 


\begin{tabular}{cccccc} 
No. Exp. & $D(\mu \mathrm{m})$ & $h_{\text {fall }}(\mathrm{mm})$ & Container & $\rho_{0}$ & Technique \\
\hline 6 & $376( \pm 32)$ & 100 & standard & $0.45( \pm 0.01)$ & camera+balance \\
1 & $376( \pm 32)$ & 150 & standard & 0.53 & camera+balance \\
1 & $1594( \pm 171)$ & 100 & standard & 0.59 & camera+balance \\
1 & $376( \pm 32)$ & N.A & small & 0.58 & NMR
\end{tabular}

Table 1: List of experiments. From left to right columns: number of experiments, particle diameter (standard deviation in bracket), free fall height for specimen generation, container type, initial packing fraction (standard deviation in bracket), and experimental technique. "standard" corresponds to cylindrical container with inner diameter $13.70 \mathrm{~mm}$ and inner height $44.22 \mathrm{~mm}$. "small" corresponds to cylindrical container with inner diameter of $6.9 \mathrm{~mm}$ and inner height of $14.5 \mathrm{~mm}$.

the signal-to-noise ratio. By means of Inverse Laplace Transform (ILT) with a procedure of non-negative least square fit to the data with Tikhonov regularization (similar to the "Contin" method as in [34, 35]), the transverse relaxation time $T_{2}$ distribution can be resolved. We refer to [32, 36, 37] for more details on NMR. Note that the 1D profile and CPMG experiments were carried out intermittently in a loop function, leading to an effective time resolution of around 9.9 minutes.

\section{Results and Discussion}

\subsection{Evaporation rate and propagation of drying front}

Fig. 2(a) shows the change in volumetric water content (and specimen weight in the inset) as a function of time for two typical experiments with initial packing fractions of 0.448 and 0.468 . For both experiments, the rate of change in weight decreases at the initial stage, entering a roughly linear stage after $t \approx 15$ hours, implying a constant drying period. Note that this observed constant drying regime is different from the one during the "Constant Rate Period" as in Fig. 1 where the latter occurs at the beginning of the drying process at high liquid content. Finally, the liquid content does not further change after about 40 hours, marking the end of the evaporation. Note that the non-zero liquid content 
Figure 2: (a) Change of volumetric water content as a function of time for two typical experiments with initial packing fractions $\rho_{0}=0.448$ and $\rho_{0}=0.468$. Inset: corresponding specimen weight as a function of time. (b) Cumulative grain size distribution of glass beads with average diameter $375.5 \mu \mathrm{m}$ (standard deviation $31.8 \mu \mathrm{m}$ ). (c) Photos of specimen taken at initial and final stages of drying. Scale bar represents $10 \mathrm{~mm}$. 
measured at the end of experiment is $0.029 \pm 0.01 \%$, which is equal to the liquid content of "dry" glass beads at laboratory condition. This is a result of adsorptive forces such that very small amount of liquid can exist at laboratory condition when no liquid is manually added into the media. Fig. 2(c) shows two photos taken at the initial and final stages of drying period, where significant settlement of grains can be seen.

Since the packing fraction is found to be strongly influenced by the interparticle forces $38-45]$, the drying-induced collapse, as explained earlier, originates from the structural instability of the loosely packed granular material once the liquid bridges evaporate, such that the assembly transitions from cohesive state to cohesionless state as the capillary force diminishes. Although it has been argued that decrease in liquid content does not result in variation in interparticle forces from liquid bridge because the decrease in liquid-solid contact area balances with increase in Laplace pressure [13, 15, 46, 47], at very low liquid content (asperity or roughness regime [46]), the capillary force decreases sharply with smaller liquid volume due to surface roughness, which has been demonstrated in force measurement experiments in liquid bridges during drying process [48 $\quad 50$ ]. This implies the existence of a critical water content at which the collapse will be triggered.

Fig. 3(a) more clearly shows that the drying rate $\dot{m}$ starts to decrease at the beginning of the experiment, entering a roughly constant rate period after 15 hours (marked by the grey region). Then $\dot{m}$ maintains at this level before decreasing to zero at the end of evaporation. To compare the observed drying rate with evaporation controlled by vapor diffusion, the inset plots $\dot{m}^{-2}$ as a function of time, where a linear relation can be observed during the first 15 hours (black-dashed line), consistent with the theory on the basis of Brutsaert 175 and Chen [1] and experiments during the "second" drying period for initially fully saturated media [9]. This confirms that the liquid bridges can be regarded as effectively isolated, and the effect from film flow is not significant.

From image analysis, as shown in Fig. 3(b), the propagation of drying front can be identified through comparing the contrast between two consecutive pho- 
Figure 3: Drying dynamics of a typical experiment. (a) Drying rate $\dot{m}$ as a function of time. Red-dotted lines mark the time of collapse event. Inset: $\dot{m}^{-2}$ as a function of time. Blackdashed line is the linear regression based on data up to $t=15$ hours. Grey area represents the transition between drying regimes. (b) Depth of drying front versus time. Inset shows the color map of difference between two consecutive images (only top region is shown for visualization purpose). (c) Height of glass beads specimen versus time. The identified collapse events are shown as blue dots. Inset: evolution of packing fraction calculated based on specimen height. Dashed line and dotted-dashed line represent the packing fractions at dry condition using pouring method (0.58) and random very loose packing (RVLP) state (0.55), respectively. Shaded area represents standard deviation. 
tos (also see Supporting Information Movie 1). It is found that the speed of receding drying front initially decreases, before entering a constant regime after $t \approx 15$ hours, in line with the transition of drying rate. After the end of drying process ( $\sim 42$ hours), the drying front cannot be identified. Fig. 3(c) shows the evolution of specimen height $h$ during drying. The changes in $h$ is found to be rather discrete, corresponding to individual collapse events. Also, it can be seen that the majority of the decreases in $h$ occurs near the extreme end of the experiment. To evaluate the packing fraction of the granular medium, the initial packing fraction can be calculated by $\phi_{0}=V_{g} / V_{c}$, with the volume of grains $V_{g}$ calculated from the total weight of dry grains divided by the density, and the volume of the container $V_{c}$ calculated from its dimension. Then, according to the photos taken during the drying process, the packing fraction at different time can be calculated by $\phi=\phi_{0} h_{0} / h$, where $h_{0}$ and $h$ are the initial and current height of the specimen determined from the image. The inset shows the corresponding packing fraction determined from images. The times of collapse events are marked as red-dashed-dotted lines in Fig. 3(a) and Fig. 3(b).

To ensure the determination of drying front from image analysis actually reflects the saturation profile within the granular medium, and shed light on the liquid distribution during the drying process, drying experiment was conducted using NMR technique under similar experimental conditions. Fig. 4(a) shows the evolution of 1-D saturation profile during NMR experiment, where light yellow color represents early stage and dark red indicates late times with 50 minutes increments. The saturation, represented by the NMR signal, is found to have similar value along the vertical direction at the start, indicating saturation homogeneity and that the medium is relatively well mixed. As the drying front recedes into the medium, the water content remains unchanged below the drying front, whereas the saturation above the drying front drops to a value smaller than the noise of NMR signal (less than $\sim 0.05 \%$ ). Fig. 4 (a) also demonstrates that the saturation gradient at the drying front is relatively sharp (around 2 $\mathrm{mm})$. It is interesting to note that, visually, the spacing between the $1 \mathrm{D}$ profiles is relatively the same, indicating a drying front propagating downwards linearly 
Figure 4: (a) Evolution of the 1-D NMR signal (direct indication of water content) profile. The transition from light yellow to dark red indicates snapshots at progressive time intervals. The time increment for each profile is 50 minutes. (b) Accumulated NMR signal versus time. The black-dashed-dotted line is added only to guide the eye. (c) Evolution of transverse relaxation time $T_{2}$ during drying experiment. Blue-dash-dotted line follows the peak value of $T_{2}$ at different times. The blue-dashed box marks the location of secondary "peak" (deviation from the gradually decreasing trend of NMR signal for smaller $T_{2}$ ). Inset: Evolution of representative $T_{2}$, calculated as the weighted average of the distribution. In all plots, blackdashed lines and black-solid lines denote the same time, i.e., 22.42 hours and 27.29 hours, respectively. 
with time, consistent with the drying regime with relatively constant drying rate. Near the end of drying, a transition can be identified (marked by the black-dashed line). After this transition, the drying region becomes stable in space, and the remaining saturation near the bottom of specimen progressively approaches the end state, which is marked by the black-solid line. Fig. 4(b) plots the accumulated NMR signal as a function of time, which is the sum of area under the curve at different times from Fig. 3(a). After an initial sharp drop, the decrease in water content again decreases roughly linearly, consistent with the previous observations. Note that the residual NMR signal after the evaporation finishes was found to be non-zero, which could result from the noise of the NMR signal itself and/or from the irreducible water content (the water content at dry state under laboratory conditions). But this should not influence the result as same signal was found for dry specimens.

\subsection{Evolution of liquid distribution}

The longitudinal relaxation time $T_{1}$, also known as the spin-lattice relaxation time, characterizes the rate at which the nuclei return to alignment with the magnetic field. The transverse relaxation time $T_{2}$, also called the spin-spin relaxation time, characterizes the time at which the transverse component of the magnetization of the nuclei comes back to the equilibrium in the magnetic field. The value of relaxation times depend on the molecular dynamic. For the liquid water in a porous medium, they depend on the pore size, the saturation, and whether the water is adsorbed on the pore surface. Information on molecular dynamics and water distribution/movement across a very wide range of length and timescales can be obtained by measurements of relaxation times [37]. Through CPMG (Carr-Purcell-Meiboom-Gill) experiment [33], the transverse relaxation time $T_{2}$ during drying can be obtained, which reads as [37, 51]:

$$
\frac{1}{T_{2}} \approx \lambda \frac{S}{V}+\frac{1}{T_{2}^{\text {bulk }}} \approx \lambda \frac{S}{V},
$$

where $\lambda$ is the surface relaxivity (unit: $\mathrm{m} / \mathrm{s}$ ), $T_{2}^{\text {bulk }}$ is the relaxation time of bulk water, $S$ is the wet surface area, and $V$ is the volume of water. The term from 
$T_{2}^{\text {bulk }}$ can be neglected in Eqn. (1) according to Tarr and Brownstein criterion [52] since the dimensionless parameter $a \lambda / D$ is found to be much less than 1, corresponding to the fast diffusion region (also denoted as surface limited relaxation), where $a$ is the characteristic size of liquid cluster, and $D \approx 2.3 \times$ $10^{-9} \mathrm{~m}^{2} \mathrm{~s}^{-1}$ is water self-diffusion coefficient at $25^{\circ} \mathrm{C}$ [53]. Fig. 44(c) shows the 245 evolution of $T_{2}$ distributions, where the arrow indicates increasing time. At early times, a peak at $T_{2} \approx 100 \mathrm{~ms}$ can be seen. If look closely, a secondary "peak" marked by the blue-dashed box (deviation from the gradually decreasing trend of NMR signal for smaller $T_{2}$ ) is also present at $T_{2} \approx 2 \mathrm{~ms}$, and these two peaks tend to merge as time increases due to the decreasing signal-to-noise ratio. Since liquid in non-saturated granular material can exist in the form of both liquid bridges between grains and thin films on grain surface [10, 12, 26, 54], it is likely that populations in the vicinities of $T_{2} \approx 100 \mathrm{~ms}$ and $T_{2} \approx 2 \mathrm{~ms}$ correspond to the liquid bridges and thin films, respectively. To test this hypothesis, for given initial volumetric water content, assuming liquid bridges contribute to the majority of liquid volume, we can estimate the average volume of liquid bridge in monodisperse spheres assuming a coordination number $N \approx 6[55$, ,56]. Then, using the relation [57]:

$$
V=0.12 d_{p}^{3} \sin ^{4}(\beta) C_{a} C_{\theta}
$$

the half-filling angle $\beta$ can be obtained, where $d_{p}$ is particle diameter, $C_{a}=1$ for particles in contact, and $C_{\theta}=1+1.1 \sin \theta$ is the correction factor for contact angle $\theta$. Finally, the volume-to-area ratio can be expressed as:

$$
\frac{V}{S}=\frac{0.12 d_{p}^{3} \sin ^{4}(\beta) C_{a} C_{\theta}}{\pi(1-\cos \beta)} .
$$

From Eqn. (3) and Eqn. (1), combined with the ratio of $T_{2}$ of two populations (2 $\mathrm{ms}$ and $100 \mathrm{~ms}$ for liquid films and liquid bridges, respectively) and the constant surface relaxivity $\lambda$ (composition of solid matrix does not change), the average thickness of thin film, which is simply calculated as $h=V / S$, is ${ }_{265}$ found to be $h \in[0.17,0.25] \mu \mathrm{m}$ for contact angle $\theta \in\left[0^{\circ}, 60^{\circ}\right]$. Past studies have indicated that the film thickness $h$ is of the same order as roughness of 
grains [58]. For example, the film thickness is found to be $h \sim 0.6 \mu \mathrm{m}$ for Fontainebleau sandstone of roughness depth $1 \mu \mathrm{m}$ [58]. Thus, for typical glass beads of roughness $\sim 0.5 \mu \mathrm{m}$ [10], the film thickness is expected to be $\sim 0.3$ $\mu \mathrm{m}$, which agrees well with the calculated value. To check the assumption that the total volume of liquid film is much smaller than that of liquid bridges, the volumetric water content under the condition where all surface areas are covered by thin film is found to be $\sim 0.2 \%$, much smaller than the total initial water content of $\sim 4.3 \%$ in NMR experiment.

Therefore, Fig. 4(c) implies that the liquid exist mainly in the form of liquid bridges (high NMR signal), and the contribution from the thin films can be effectively ignored. The trajectory of $T_{2}$ with maximum NMR signal is shown as blue-dot-dashed line. It remains almost constant before the transition marked by black-dashed line, then experiencing a sharp decrease. This is indeed consistent with the previous observation where the drying front recedes into the porous medium, leading to a significant decrease in total volume of liquid (corresponding to decrease in NMR signal), but with no significant change of the geometrical feature of liquid clusters (corresponding to constant $T_{2}$ ). The latter is mainly represented by the volume-to-area ratio of the liquid bridges that make up the bulk of liquid volumes (those below the drying front). The volumeto-area ratio only starts to decrease near the end of drying, corresponding to a drying region that is stable in space (Fig. 4(a) after the black-dashed line), during which the representative volume of liquid bridges decreases. Similar tendency can be reflected from the representative $T_{2}$ (Inset of Fig. 4 (c)), calculated as the weighted average of $T_{2}$ distribution at different times, where it decreases slowly before $t=22.4 \mathrm{~h}$ due to the weighted averaging process, and a sharp decrease can be observed after the transition. It is interesting to note that the spatial shift of saturation profile (front propagation) in Fig. 4(a) corresponds to the decrease in signal intensity of $T_{2}$ distribution in Fig. 4(c), whereas a spatially stable decrease in saturation after the transition $(t=22.4 \mathrm{~h})$ corresponds to the shift in value of representative $T_{2}$. These observations together reveal the global and local evolution of volume and shape of liquid clusters. 


\subsection{Drying-induced collapse}

Given the observation of the drying front and sharp saturation gradient

To ensure the repeatability of the experiments and gain sufficient statistical information on drying-induced collapse, the specimen height versus time from 
Figure 5: (a) Height versus time for six repeated experiments. Concentrated collapse events are observed near the end of drying ( $t \approx 40$ hours). (b) Corresponding packing fraction determined from the image analysis. Solid line and dotted-dashed line represent the packing fractions at dry condition using pouring method (0.58) and random very loose packing (RVLP) state (0.55), respectively. (c) Typical data of the sum in the horizontal direction of difference of two consecutive images right before $\left(c_{n}-1\right)$, at $\left(c_{n}\right)$, and right after $\left(c_{n}+1\right)$ a collapse event. Distance between the black-dashed lines represents the individual collapse region length $l_{c}$. Inset: photos right before/after a collapse event. The region above the white-dashed line collapses, whereas the region below remains unaffected. (d) Statistics of collapse events for six experiments: change in height $\Delta h$ versus individual collapse region length $l_{c}$. Filled and hollow symbols represent the first and subsequent collapse events, respectively. Error bars represent the standard deviation of surface profile fluctuation. Black-dashed line shows the theory when packing fraction changes from 0.45 to 0.58 homogeneously. 
six experiments are plotted in Fig. 5(a). Again, concentrated collapses are observed at $t \approx 40$ hours depending on initial water content and minor fluctuations in relative humidity and temperature, and almost no collapse event occurs in the first 30 hours even with significant apparent dry region after propagation of drying front (Fig. 3(c)). The corresponding packing fraction $\rho$ calculated from

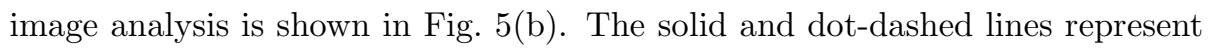
the packing fractions measured at dry condition using pouring method 19] and lower limit of random very loose packing (RVLP) 20 23], respectively. As can be seen in Fig. 5(b), the packing fraction increases from $\sim 0.45$ to $\sim 0.55$ after collapse, close to the RVLP. Note that there is an underestimation of the packing fraction, as the surface is no longer perfectly smooth after collapse such that the occupied volumes of particles determined from 2D images are typically greater than actual ones. This may explain some of the final packing fractions are still smaller than the RVLP limit.

Apart from the identification of drying front and collapse height, the individual collapse region, characterized by its length $l_{c}$ can also be obtained from the image analysis. Firstly, the image contrast (subtraction of image matrix) between every two consecutive images is calculated. Then, the cumulative image contrast along the horizontal direction is calculated, giving an array along the vertical direction. If no collapse event occurs during the time between these two images, the cumulative contrast array should be mainly noise with relatively small values. If, however, a collapse event happens, the image contrast array should contain greater values since there is significant difference between two consecutive images. Fig. 5 (c) shows the image contrast array before $\left(c_{n}-1\right)$, at $\left(c_{n}\right)$, and after $\left(c_{n}+1\right)$ an individual collapse event. Three distinct regions can be identified: (1) large contrast region with cumulative horizontal contrast above 2000, which represents the region that is filled before the collapse, but empty after; (2) moderate contrast region with contrast smaller than 2000 and greater than 300, which represents the region that is filled with initial packing state, but collapses into different (denser) packing state after; (3) small contrast region with contrast less than 300 , where there is no change in structure and the 
fluctuations are mainly from image noise. Note that the changes in image contrast at the boundaries of different regions are relatively sharp, and it is found that minor variations in these empirically selected threshold values do not significantly impact the results. In the case of Fig. 5(c), the distance between the black-dashed lines is thus $l_{c}$. Note that the individual collapse region length $l_{c}$ is calculated as the effective one, i.e, it only represents the region that evolves from loose state towards denser state at each individual collapse, without the cumulative effect from previous collapses. The inset shows the photos before and after an initial collapse event, where the region below the white-dashed line remain unaffected. This suggests that even in the apparent dry region, there exists a saturation gradient, which may correspond to a third drying front that separates the specimen by the effectiveness of the capillary bridge through a critical water content. The region below this drying front can withstand the impact of collapse above it and remain intact due to the capillary force. As one of the main findings, this third drying front has not been discovered before.

375 However, a more thorough investigation requires higher resolution of measuring device and more controlled experimental condition, which is beyond the scope of current work.

Fig. 5(d) depicts the correlation between change in height and individual collapse region length. Filled and hollow circles represent initial and subsequent collapse events for each experiment in different colors. Error bars represent the standard deviation of surface profile fluctuations from image analysis. It shows that the initial collapse events tend to have greater region of impact. In addition, most points can be described by a theoretical line assuming the packing fraction changes from 0.45 to 0.58 for each individual collapse event (see appendix for calculation of this line). This again indicates that the nature of collapse is local. Besides, it shows that both the initial packing structure before collapse, and the change in packing fraction for each individual collapse event, are relatively homogeneous. 


\subsection{Transition in drying regimes}

One possible reason that results in the observed transition in drying regimes, i.e., the decreasing rate regime to constant rate regime indicated by the grey region in Fig. 3 (a), could be the theory of enhanced vapour diffusion in nonsaturated porous media [64, 65], i.e., in the presence of thermal or capillary gradient, the vapor can condensate on one side of liquid island and subsequently evaporate from the other side, which reduces the effective diffusion path length. Nevertheless, the enhancement factor in this model decreases with decreasing saturation, being close to one at low saturation [65]. Since the initial water content is also low in the current study $(\sim 2 \%)$, and the transition takes place in later stage of drying (at even lower water content), the enhanced diffusion theory alone cannot explain the observed results.

Besides, the effect from film flow at low water content regime has been explored in the recent years [25 28, 54, 63], where the liquid connectivity is maintained through the thin liquid film at the grain surface. As a result, the drying flux through the film flow is proportional to the specific surface area of the granular medium, which implies that the drying flux at transition in drying regimes, if mainly transported through film flow, should be sensitive to the grain size. However, we did not observe this phenomenon and the critical drying rate at which the transition occurs is similar for $376 \pm 32 \mu \mathrm{m}$ and $1594 \pm 171 \mu \mathrm{m}$ glass beads (Fig. A.1 in the Appendix).

Actually, it is likely that the observed phenomena is due to Kelvin effect. The Kelvin equation $\ln p^{*}=\gamma V_{m} / r R T$ indicates that the relative vapor pressure at the liquid-gas interface $p^{*}$ depends on the capillary pressure $\gamma / r$, with $\gamma$ surface tension, $V_{m}$ molar volume, $r$ the radius of curvature of liquid-gas interface, $R$ universal gas constant, and $T$ temperature. As the drying front propagates and the region of the porous medium above it approaches dry condition, the curvature of liquid bridges above the drying front quickly increases (as their volume decreases), causing a drop in vapor pressure at the surface of liquid bridges and slowing down the drying process of those very small liquid bridges in the apparent dry region. Thus, the Kelvin effect explains the delayed evap- 
oration of small liquid bridges that are responsible for supporting the loosely packed granular materials, leading to the observation that the occurrences of most collapse events are concentrated near the very end of the whole drying process. As the evaporation proceeds, the overall drying rate decreases due to greater diffusion distance according to Fick's law, whereas a greater number of menisci are exposed to evaporation, thus leading to a deviation from the theory where Kelvin effect (and thus the presence of liquids above the drying front) is not considered. The enhancement in drying rate due to Kelvin effect is also reported in a recent work using pore-network simulation [66]. It is worth noting that this phenomenon can be regarded as an equilibration process [8]. However, different from capillary flow where the equilibration takes place within liquid phase due to difference in capillary pressure, it is due to constrain in the vapor pressure according to Kelvin equation. Although without detailed discussion, similar drying enhancement phenomenon at low saturation (less than 5\%) is also present in the study by Thiery et al [8]. In fact, the Kelvin effect can be significant either for very fine particles or very low water content [8, 67], and the current study demonstrates for the first time the drying enhancement phenomenon due to Kelvin effect for grains with size of hundreds of micrometers.

In this study, glass beads were chosen as the model granular materials for the drying experiment. With careful choice of the particle diameter accord${ }_{440}$ ing to previous studies [11, 19], the effect from van der Waals forces can be effectively excluded, and the loosely packed wet granular assembly with packing fraction significantly less than the one at cohesionless state $(0.58$ in this case) can be generated. For other granular materials, however, depending on the initial state of the granular assembly and particle properties, different behaviour can be expected. For example, for finer particles with other cohesive forces apart from capillary force, the resulting packing fraction after collapse due to disappearance of liquid bridges can be some intermediate value between the initial and cohesionless state, depending on the relative significance of the cohesive force to the gravity $38-45]$. Besides, for grains with greater surface roughness, on the one hand, enhanced structural stability might be expected due 
to greater friction coefficient. On the other hand, the effectiveness of the small liquid bridges might decrease, i.e., increase in surface roughness is associated with smaller capillary force from small liquid bridges as a result of the asperity or roughness regime [46], which can consequently lead to an earlier transition towards cohesionless (or less cohesive) state during the drying process. These competing mechanisms originated from change in surface roughness, and other factors such as particle shape and grain size distribution, are worthy of further investigation. In addition, the experimental condition at which the evaporation process occurs is also likely to impact the drying behaviour. The drying rate in the current study is relatively slow, i.e., it takes about 2 days for the drying of granular materials with $2 \%$ initial water content to complete under standard laboratory condition. With faster drying rate due to higher temperature, lower relative humidity, or imposed external airflow in the environment, the influence of Kelvin effect on the drying rate may become less significant even near the end of drying process. The aforementioned aspects, as well as more systematic and quantitative characterization of the drying process with the help of more accurate equipment and thorough control of experimental condition, are some examples of future research directions.

\section{Conclusions}

The drying of loosely packed granular materials from low initial water content was experimentally studied. The drying rate is found to decrease from the beginning, corresponding to the decreasing rate period controlled by vapor diffusion, which is associated with a propagation of drying front with a sharp saturation gradient identified through image analysis and magnetic resonance imaging. Then, a deviation in drying rate from diffusion limited evaporation is observed. The enhancement of the evaporation at the later stage of drying process is attributed to Kelvin effect.

We also observed the drying-induced collapse of granular medium due to its structural instability as the liquid bridges evaporate and capillary forces 

$V_{1}=A l_{c}$, with $A$ the cross-section area, $l_{c}$ the height (also the collapse region length). Let $\phi_{1}$ be the packing fraction before collapse, leading to the volume

of liquid in the form of liquid bridges in the apparent dry region due to Kelvin effect, which can be further extracted near the end of drying process. Through analyzing the collapse statistics, it is found that collapse event is local in space and time, which implies that, apart from previously identified primary and secondary drying fronts, there exists a third drying front in the apparent dry region, which propagates relatively quickly at the end of drying.

\section{Acknowledgments}

This work was financially supported by Australian Research Council (Projects DP170102886) and The University of Sydney SOAR Fellowship. YG acknowledges the financial support of Labex MMCD(ANR-11-LABX-022-01) for his stay at Laboratoire Navier at ENPC. ZW thanks Baptiste Chabot, Xavier Boulay, Loc Lesueur, and Emmanuel De Laure for the assistance on experiments. Data associated with this work are available online (https://doi.org/10.6084/m9.figshare.14368688).

\section{Appendix}

Drying curves at different initial packing fractions and grain size.

Fig. A.1 shows the drying curves (evolution of specimen weight, drying rate $\dot{m}$, and $\dot{m}^{-2}$ ) for grains at different initial packing fractions (a-c) and larger grain size (d). The black-solid lines are linear fittings based on data at early stages of drying, which represent the theory predicted based on Fick's law. An enhancement in drying rate can be observed in all experiments, which corresponds to a downward deviation from the linear relation. The drying rates at which this transition occurs are $\sim 3 \mathrm{~g} / \mathrm{min}$ for all cases (as in $\dot{m}$ - $t$ plots).

Determination of theoretical line relating $l_{c}$ and $\Delta h$. The line can be determined based on the idea of conservation of volume, i.e., volume occupied ${ }_{505}$ by the grains remain the same. For a given volume before collapse, the volume 
Figure A.1: Drying curves of unsaturated porous media. (a-c) Glass beads of diameter 375.5 \pm 31.8 and different initial packing fractions. (d) Glass beads of diameter $1594 \pm 171$. Solid lines are linear fittings using data at the early stages. 
occupied by grains $V_{g}=\phi_{1} V_{1}$. After collapse, if the packing fraction is $\phi_{2}, V_{g}=$ $\phi_{2} V_{2}$, with $V_{2}=A\left(l_{c}-\Delta h\right)$ the volume after collapse. So, $V_{g}=\phi_{1} V_{1}=\phi_{2} V_{2}$,

i.e., $\phi_{1} A l_{c}=\phi_{2} A\left(l_{c}-\Delta h\right)$, in other words, $\Delta h=l_{c}\left(1-\phi_{1} / \phi_{2}\right)$.

[1] W. Brutsaert, D. Chen, Desorption and the two stages of drying of natural tallgrass prairie,

Water Resources Research 31 (5) (1995) 1305-1313. arXiv:https://agupubs.onlinelibrary.wiley.com/doi/pdf/10.1029/95WR00323, doi:https://doi.org/10.1029/95WR00323

URL https://agupubs . onlinelibrary.wiley.com/doi/abs/10.1029/95WR00323

[2] P. Coussot, Scaling approach of the convective drying of a porous medium, The European Physical Journal B - Condensed Matter and Complex Systems 15 (3) (2000) 557-566. doi:10.1007/s100510051160.

URL https://doi.org/10.1007/s100510051160

[3] D. Or, P. Lehmann, E. Shahraeeni, N. Shokri, Advances in Soil Evaporation PhysicsA Review, Va$\begin{array}{lllll}\text { dose } & \text { Zone } & \text { Journal } & 12 & \text { (4), }\end{array}$ arXiv:https://pubs.geoscienceworld.org/vzj/article-pdf/12/4/vzj2012.0163/2983949/vzj201 doi:10.2136/vzj2012.0163.

URL https://doi.org/10.2136/vzj2012.0163

[4] N. Shokri, P. Lehmann, D. Or, Critical evaluation of enhancement factors for vapor transport through uns Water Resources Research 45 (10).arXiv: https://agupubs.onlinelibrary .wiley.com/doi/pdf/10.10 doi:https://doi.org/10.1029/2009WR007769.

URL https://agupubs .onlinelibrary.wiley.com/doi/abs/10.1029/2009WR007769

[5] P. Lehmann, S. Assouline, D. Or, Characteristic lengths affecting evaporative drying of porous media, Phys. Rev. E 77 (2008) 056309. doi:10.1103/PhysRevE.77.056309.

URL https://link.aps.org/doi/10.1103/PhysRevE.77.056309

[6] A. G. Yiotis, D. Salin, E. S. Tajer, Y. C. Yortsos, Analytical solutions of drying in porous media for gravity-stabilized fronts, Phys. Rev. E 85 (2012) 046308. doi:10.1103/PhysRevE.85.046308. URL https://link.aps .org/doi/10.1103/PhysRevE.85.046308 
[7] A. G. Yiotis, D. Salin, E. S. Tajer, Y. C. Yortsos, Drying in porous media with gravity-stabilized fronts: Experimental results,

Phys. Rev. E 86 (2012) 026310. doi:10.1103/PhysRevE.86.026310.

URL https://link.aps .org/doi/10.1103/PhysRevE.86.026310

[8] J. Thiery, S. Rodts, D. A. Weitz, P. Coussot, Drying regimes in homogeneous porous media from macro- to nanoscale, Phys. Rev. Fluids 2 (2017) 074201. doi:10.1103/PhysRevFluids.2.074201. URL https://link.aps.org/doi/10.1103/PhysRevFluids.2.074201

[9] N. Shokri, D. Or, What determines drying rates at the onset of diffusion controlled stage-2 evaporation fr Water Resources Research 47 (9). arXiv:https://agupubs.onlinelibrary.wiley.com/doi/pdf/10.102 doi:https://doi.org/10.1029/2010WR010284. URL https://agupubs . onlinelibrary.wiley.com/doi/abs/10.1029/2010WR010284

[10] Z. Fournier, D. Geromichalos, S. Herminghaus, M. M. Kohonen, F. Mugele, M. Scheel, M. Schulz, B. Schulz, C. Schier, R. Seemann, A. Skudelny, Mechanical properties of wet granular materials, Journal of Physics: Condensed Matter 17 (9) (2005) S477-S502.

[11] V.-D. Than, P. Aimedieu, J.-M. Pereira, J.-N. Roux, M. Bornert, A.-M. Tang, Macro-microscopic one-dimensional compression of wet granular soils by experimental investigation, E3S Web of Conferences 9 (2016) 06001.

[12] M. Scheel, R. Seemann, M. Brinkmann, M. Di Michiel, A. Sheppard, B. Breidenbach, S. Herminghaus, Morphological clues to wet granular pilestability, Nature materials 7 (2008) 189-93.

[13] Y. I. Rabinovich, M. S. Esayanur, B. M. Moudgil, Capillary forces between two spheres with a fixed volume liquid bridge: Theory and experiment, Langmuir 21 (24) (2005) 10992-10997, pMID: 16285763.

[14] M. Scheel, R. Seemann, M. Brinkmann, M. D. Michiel, A. Sheppard, S. Herminghaus, Liquid distribution and cohesion in wet granular assemblies be- 
yond the capillary bridge regime, Journal of Physics: Condensed Matter $20(49)$ (2008) 494236.

[15] H.-J. Butt, M. Kappl, Normal capillary forces, Advances in Colloid and Interface Science 146 (1) (2009) 48-60.

[16] C. Feng, A. Yu, Effect of liquid addition on the packing of mono-sized coarse spheres, Powder Technology 99 (1) (1998) 22-28.

[17] R. Y. Yang, R. P. Zou, A. B. Yu, Numerical study of the packing of wet coarse uniform spheres, AIChE Journal 49 (7) (2003) 1656-1666.

[18] N. Mitarai, F. Nori, Wet granular materials, Advances in Physics 55 (1-2) (2006) $1-45$.

[19] Z. Wang, J.-M. Pereira, Y. Gan, Packing of wet monodisperse spheres $\begin{array}{lllll}\text { Powder } & \text { Technology } & 378 & \text { (2021) }\end{array}$ doi:https://doi.org/10.1016/j.powtec.2020.09.074 URL http://wWw.sciencedirect.com/science/article/pii/S0032591020309384

[20] G. Y. Onoda, E. G. Liniger, Random loose packings of uniform spheres and the dilatancy onset, Phys. Rev. Lett. 64 (1990) 2727-2730.

[21] M. P. Ciamarra, A. Coniglio, Random very loose packings, Phys. Rev. Lett. $101(2008) 128001$.

[22] M. Jerkins, M. Schröter, H. L. Swinney, T. J. Senden, M. Saadatfar, T. Aste, Onset of mechanical stability in random packings of frictional spheres, Phys. Rev. Lett. 101 (2008) 018301.

[23] G. R. Farrell, K. M. Martini, N. Menon, Loose packings of frictional spheres, Soft Matter 6 (2010) 2925-2930.

[24] T. T. Vo, S. Nezamabadi, P. Mutabaruka, J.-Y. Delenne, F. Radjai, Additive rheology of complex granular flows, Nature Communications 11 (1) (2020) 1476. doi:10.1038/s41467-020-15263-3 URL https://doi .org/10.1038/s41467-020-15263-3 
[25] M. Tuller, D. Or, Hydraulic conductivity of variably saturated porous media: Film and corner flow in ang Water Resources Research $37 \quad$ (5) (2001) 1257-1276. arXiv:https://agupubs.onlinelibrary.wiley.com/doi/pdf/10.1029/2000WR900328 doi:https://doi.org/10.1029/2000WR900328. URL https : //agupubs . onlinelibrary . wiley.com/doi/abs/10.1029/2000WR900328

[26] M. Tuller, D. Or, Water films and scaling of soil characteristic curves at low water contents, Water Resources Research 41 (9). arXiv:https://agupubs . onlinelibrary.wiley.com/doi/pdf/10.102 doi:https://doi.org/10.1029/2005WR004142. URL https://agupubs .onlinelibrary.wiley.com/doi/abs/10.1029/2005WR004142

[27] Y. Wang, J. Ma, Y. Zhang, M. Zhao, W. M. Edmunds,

A new theoretical model accounting for film flow in unsaturated porous media,

Water Resources Research $49 \quad$ (8) (2013) 5021-5028. arXiv:https://agupubs.onlinelibrary.wiley.com/doi/pdf/10.1002/wrcr.20390 doi:https://doi.org/10.1002/wrcr.20390 URL https://agupubs.onlinelibrary.wiley.com/doi/abs/10.1002/wrcr.20390

[28] Y. Wang, M. Jin, Z. Deng, Alternative model for predicting soil hydraulic conductivity over the complete Water Resources Research 54 (9) (2018) 6860-6876. arXiv:https://agupubs.onlinelibrary.wiley.com/doi/pdf/10.1029/2018WR023037, doi:https://doi.org/10.1029/2018WR023037. URL https://agupubs.onlinelibrary.wiley.com/doi/abs/10.1029/2018WR023037

[29] J.-F. Bruchon, J.-M. Pereira, M. damme, N. Lenoir, P. Delage, M. Bornert, Full 3d investigation and characterisation of capillary collapse of a loose unsaturated sand using x-ray ct $\begin{array}{llllll}\text { Granular } & \text { Matter } & 15 & \text { (6) }\end{array}$ doi:10.1007/s10035-013-0452-6. URL https://doi.org/10.1007/s10035-013-0452-6

[30] L. Chiapponi, Water retention curves of multicomponent mixtures of spherical particles Powder Technology 320 (2017) 646-655. 
doi:https://doi.org/10.1016/j.powtec.2017.07.083

[31] N. Ben Abdelouahab, A. Gossard, S. Rodts, B. Coasne, P. Coussot, Convective drying of a porous medium with a paste cover, The European Physical Journal E 42 (5) (2019) 66. doi:10.1140/epje/i2019-11829-4. URL https://doi .org/10.1140/epje/i2019-11829-4

[32] P. Coussot, Progress in rheology and hydrodynamics allowed by nmr or mri techniques,

$\begin{array}{lllllll}\text { Experiments in } & \text { Fluids } & 61 & (9) & \text { (2020) }\end{array}$

doi:10.1007/s00348-020-03037-y

URL https://doi.org/10.1007/s00348-020-03037-y

[33] S. Meiboom, D. Gill, Modified Spin-Echo Method for Measuring Nuclear Relaxation Times, Review of Scientific Instruments 29 (8) (1958) 688-691. doi:10.1063/1.1716296.

[34] S. W. Provencher, A constrained regularization method for inverting data represented by linear algebraic Computer Physics Communications $27 \quad$ (3) (1982) 213-227. doi:https://doi.org/10.1016/0010-4655(82)90173-4. URL/http://www.sciencedirect.com/science/article/pii/0010465582901734

[35] K. P. Whittall, A. L. MacKay, Quantitative interpretation of nmr relaxation data, Journal of Magnetic Resonance (1969) 84 (1) (1989) 134-152. doi:https://doi.org/10.1016/0022-2364(89)90011-5. URL http://www.sciencedirect.com/science/article/pii/0022236489900115

[36] P. F. Faure, S. Rodts, Proton nmr relaxation as a probe for setting cement pastes, Magnetic Resonance Imaging $26 \quad$ (8) (2008) 1183-1196. doi:https://doi.org/10.1016/j.mri.2008.01.026. URL http://wWw.sciencedirect.com/science/article/pii/S0730725X08000520

[37] A. Valori, P. McDonald, K. Scrivener, The morphology of csh: Lessons from 1h nuclear magnetic resonance relaxometry,

Cement and Concrete Research 49 (2013) 65-81. 
doi:https://doi.org/10.1016/j.cemconres.2013.03.011.

URL http://wWw.sciencedirect.com/science/article/pii/S0008884613000653

[38] R. Y. Yang, R. P. Zou, A. B. Yu, Computer simulation of the packing of fine particles, Phys. Rev. E 62 (2000) 3900-3908.

[39] A. Yu, C. Feng, R. Zou, R. Yang, On the relationship between porosity and interparticle forces, Powder Technology 130 (1) (2003) 70-76.

[40] J. Blum, R. Schräpler, Structure and mechanical properties of high-porosity macroscopic agglomerates formed by random ballistic deposition, Phys. Rev. Lett. 93 (2004) 115503.

[41] D. Lohse, R. Rauh, R. Bergmann, D. Meer, Creating a dry variety of quicksand, Nature 432 (2005) 689-90.

[42] K. J. Dong, R. Y. Yang, R. P. Zou, A. B. Yu, Role of interparticle forces in the formation of random loose packing, Phys. Rev. Lett. 96 (2006) 145505.

[43] P. B. Umbanhowar, D. I. Goldman, Low density fragile states in cohesive powders, American Journal of Physics 74 (8) (2006) 720-721.

[44] R. Yang, R. Zou, K. Dong, X. An, A. Yu, Simulation of the packing of cohesive particles, Computer Physics Communications 177 (1) (2007) 206209, proceedings of the Conference on Computational Physics 2006.

[45] E. Parteli, J. Schmidt, C. Blmel, K.-E. Wirth, W. Peukert, T. Pschel, Attractive particle interaction forces and packing density of fine glass powders, Scientific reports 4 (2014) 6227.

[46] T. C. Halsey, A. J. Levine, How sandcastles fall, Phys. Rev. Lett. 80 (1998) $3141-3144$.

[47] H.-J. Butt, Capillary forces: Influence of roughness and heterogeneity, Langmuir 24 (9) (2008) 4715-4721, pMID: 18442225. 
[48] B. Mielniczuk, T. Hueckel, M. S. E. Youssoufi, Evaporation-induced evolution of the capillary force between two grains

$\begin{array}{lllll}\text { Granular } & \text { Matter } & 16 & \text { (5) } \quad \text { (2014) }\end{array}$

[49] B. Mielniczuk, T. Hueckel, M. S. El Youssoufi, Laplace pressure evolution and four instabilities in evaporating two-grain liquid bridges, Powder Technology $283 \quad$ (2015) 137-151. doi:https://doi.org/10.1016/j.powtec.2015.05.024 URL http://www.sciencedirect.com/science/article/pii/S0032591015004106

[50] T. Hueckel, B. Mielniczuk, M. S. El Youssoufi, Adhesion-force micro-scale study of desiccating granular material,
Gotechnique
70
(12)
(2020)
$1133-1144$.

arXiv:https://doi.org/10.1680/jgeot.18.P.298

doi:10.1680/jgeot.18.P.298.

URL https://doi .org/10.1680/jgeot.18.P.298

[51] R. M. E. Valckenborg, L. Pel, K. Hazrati, K. Kopinga, J. Marchand, Pore water distribution in mortar during drying as determined by $\mathrm{nmr}$,

Materials and Structures $34 \quad$ (10) (2001) 599-604.

doi:10.1007/BF02482126.

URL https : //doi .org/10.1007/BF02482126

[52] K. R. Brownstein, C. E. Tarr, Importance of classical diffusion in $\mathrm{nmr}$ studies of water in biological cells, Phys. Rev. A 19 (1979) 2446-2453. doi:10.1103/PhysRevA.19.2446. URL https://link.aps.org/doi/10.1103/PhysRevA.19.2446

[53] M. Holz, S. R. Heil, A. Sacco, Temperature-dependent self-diffusion coefficients of water and six selected $\mathrm{n}$ Phys. Chem. Chem. Phys. 2 (2000) 4740-4742. doi:10.1039/B005319H

URL http://dx.doi.org/10.1039/B005319H

[54] Y. Wang, J. Ma, H. Guan, A mathematically continuous model for describing the hydraulic properties of Journal of Hydrology 541 (2016) 873-888. 
doi:https://doi.org/10.1016/j.jhydrol.2016.07.046

URL http://www.sciencedirect.com/science/article/pii/S0022169416304760

[55] L. Liu, Z. Zhang, A. Yu, Dynamic simulation of the centripetal packing of mono-sized spheres, Physica A: Statistical Mechanics and its Applications 268 (3) (1999) 433-453.

[56] W. Fei, G. A. Narsilio, Impact of three-dimensional sphericity and roundness on coordination number, Journal of Geotechnical and Geoenvironmental Engineering 146 (12) (2020) 06020025.

[57] T. Weigert, S. Ripperger, Calculation of the liquid bridge volume and bulk saturation from the half-filling angle, Particle \& Particle Systems Characterization 16 (5) (1999) 238-242.

[58] M. Han, S. Youssef, E. Rosenberg, M. Fleury, P. Levitz, Deviation from archie's law in partially saturated porous media: Wetting film versus disconnectedness of Phys. Rev. E 79 (2009) 031127. doi:10.1103/PhysRevE.79.031127. URL https://link.aps.org/doi/10.1103/PhysRevE.79.031127

[59] G. C. Cho, J. C. Santamarina, Unsaturated particulate materials\&\#x2014;particle-level studies, Journal of Geotechnical and Geoenvironmental Engineering 127 (1) (2001) 84-96. doi:10.1061/(ASCE) 1090-0241(2001) 127:1(84).

[60] S. Wu, D. H. Gray, F. E. Richart, Capillary effects on dynamic modulus of sands and silts, Journal of Geotechnical Engineering 110 (9) (1984) 11881203. doi:10.1061/(ASCE) 0733-9410(1984)110:9(1188)

[61] R.-P. Zou, C.-L. Feng, A.-B. Yu, Packing density of binary mixtures of wet spheres, Journal of the American Ceramic Society 84 (3) (2001) 504-508. arXiv:https://ceramics.onlinelibrary.wiley.com/doi/pdf/10.1111/j.1151-2916.2001.tb00690 doi:10.1111/j.1151-2916.2001.tb00690.x

URL https: //ceramics.onlinelibrary.wiley.com/doi/abs/10.1111/j.1151-2916.2001.tb00690.x 
[62] R. Zou, J. Xu, C. Feng, A. Yu, S. Johnston, N. Standish, $\quad$ Packing of multi-sized mixtures of wet coarse spheres,

doi:https://doi.org/10.1016/S0032-5910(02)00229-2

URL http://www.sciencedirect.com/science/article/pii/S0032591002002292

[63] Y. Wang, O. Merlin, G. Zhu, K. Zhang,

A physically based method for soil evaporation estimation by revisiting the soil drying process

Water Resources Research 55 (11) (2019) 9092-9110.

arXiv:https://agupubs.onlinelibrary.wiley.com/doi/pdf/10.1029/2019WR025003

doi:https://doi.org/10.1029/2019WR025003.

URL https://agupubs .onlinelibrary.wiley.com/doi/abs/10.1029/2019WR025003

[64] S. W. Webb, C. K. Ho, Review of enhanced vapor diffusion in porous media,

Tech. rep., United States, sAND-98-1819C (1998).

URL http://inis.iaea.org/search/search.aspx?orig_q=RN : 30009514

[65] E. Shahraeeni, D. Or, Pore scale mechanisms for enhanced vapor transport through partially saturated pc Water Resources Research 48 (5). arXiv:https://agupubs . onlinelibrary.wiley.com/doi/pdf/10.102 doi:https://doi.org/10.1029/2011WR011036.

URL https: //agupubs . onlinelibrary.wiley.com/doi/abs/10.1029/2011WR011036

[66] O. Maalal, M. Prat, D. Lasseux, Pore network model of drying with kelvin effect,
Physics
of Fluids
33
(2)
(2021)
027103.

arXiv:https://doi.org/10.1063/5.0035651, doi:10.1063/5.0035651.

URL https://doi.org/10.1063/5.0035651

[67] Q. Yang, P. Z. Sun, L. Fumagalli, Y. V. Stebunov, S. J. Haigh, Z. W. Zhou, I. V. Grigorieva, F. C. Wang, A. K. Geim, Capillary condensation under atomic-scale confinement, Nature 588 (7837)

(2020) 250-253. doi:10.1038/s41586-020-2978-1.

URL https://doi.org/10.1038/s41586-020-2978-1 
Figure 1. 


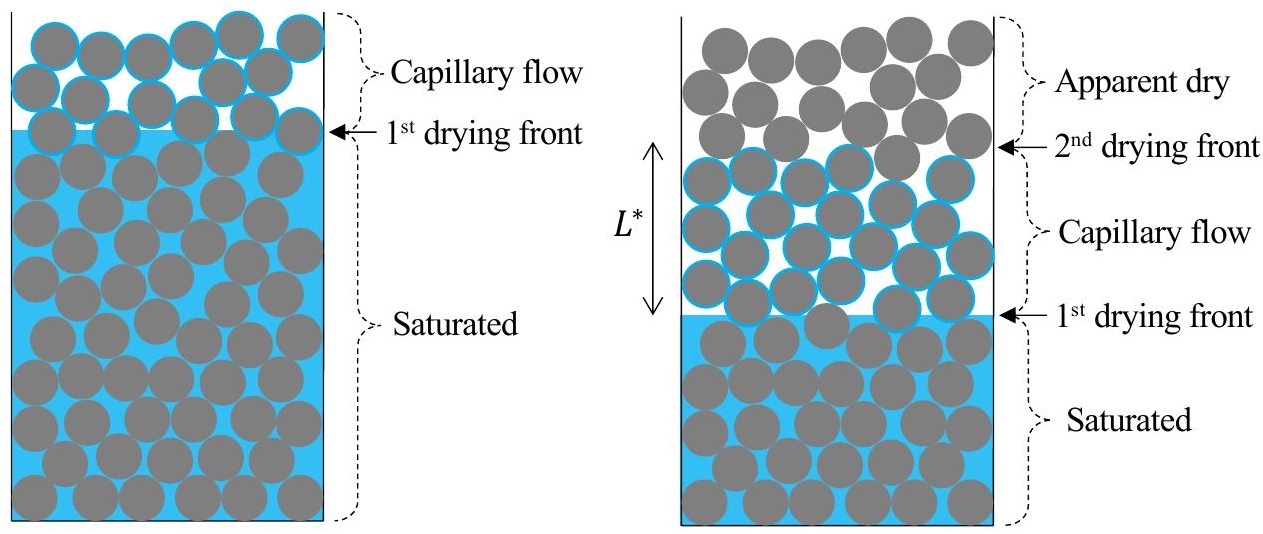


Figure 2. 

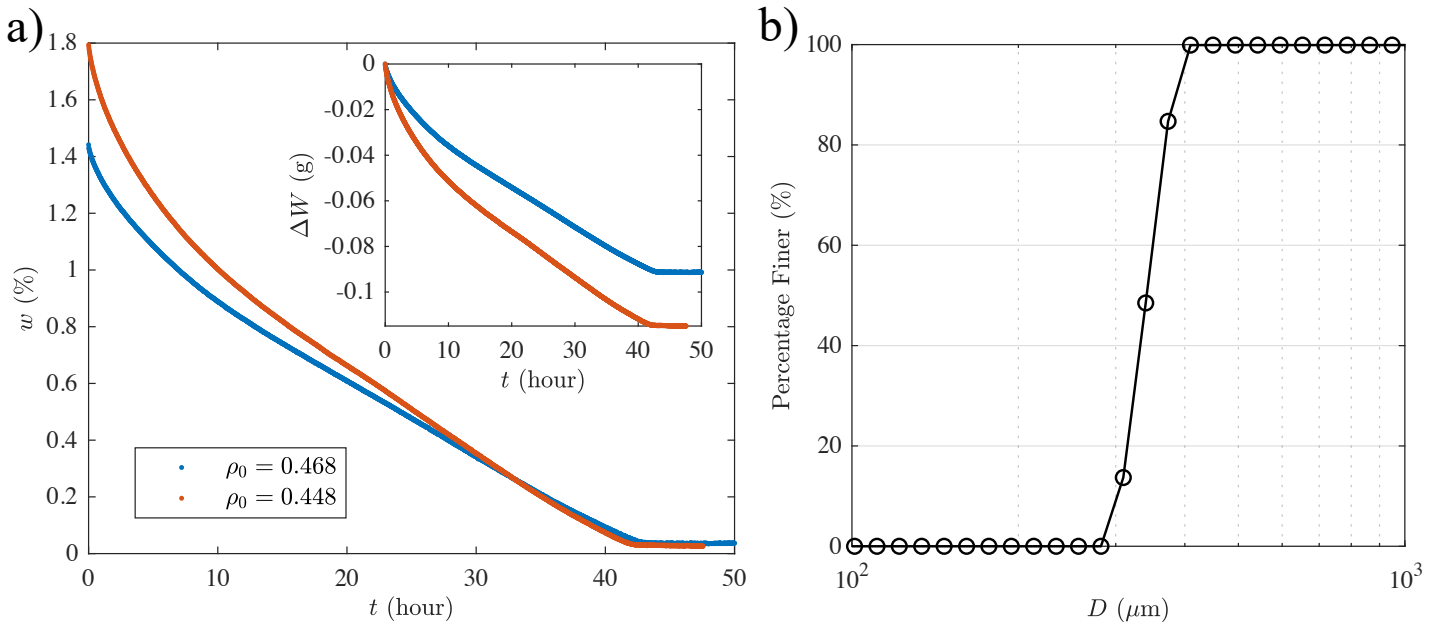

c) 
Figure 3. 
Figure 4. 
Figure 5. 
Figure A1. 
